PROCEEDINGS OF THE

AMERICAN MATHEMATICAL SOCIETY

Volume 134, Number 9, September 2006, Pages 2767-2770

S $0002-9939(06) 08257-8$

Article electronically published on March 21, 2006

\title{
BOUNDARY STRUCTURE OF HYPERBOLIC 3-MANIFOLDS ADMITTING ANNULAR FILLINGS AT LARGE DISTANCE
}

\author{
SANGYOP LEE
}

(Communicated by Ronald A. Fintushel)

\begin{abstract}
We show that if a hyperbolic 3-manifold $M$ with $\partial M$ a union of tori admits two annular Dehn fillings at distance $\Delta \geq 3$, then $M$ is bounded by at most three tori.
\end{abstract}

An annulus or torus embedded in a 3-manifold is essential if it is incompressible, boundary-incompressible and is not boundary-parallel. A 3-manifold is annular (resp. toroidal) if it contains an essential annulus (resp. torus). Otherwise, it is anannular (resp. atoroidal). Also, a 3-manifold is irreducible if any embedded 2-sphere bounds an embedded 3-ball, and is boundary-irreducible if its boundary is incompressible. Thurston 9] has shown that a compact, orientable 3-manifold $M$ with non-empty boundary is irreducible, boundary-irreducible, atoroidal and anannular if and only if it is hyperbolic, in the sense that $M$ with its boundary tori removed admits complete finite volume hyperbolic structure with totally geodesic boundary.

Let $M$ be a compact, connected, orientable 3-manifold with torus boundary component $T_{0}$. The Dehn filling of $M$ with slope $\gamma$ is the manifold $M(\gamma)$ obtained by attaching a solid torus $V_{\gamma}$ to $M$ along their boundary so that a meridian of $V_{\gamma}$ is identified with a curve of slope $\gamma$ on $T_{0}$. For two slopes $\gamma_{1}, \gamma_{2}$ on $T_{0}, \Delta\left(\gamma_{1}, \gamma_{2}\right)$ denotes their minimal geometric intersection number and is called a distance between the slopes.

Suppose that given a hyperbolic 3-manifold $M$, there are two slopes $\gamma_{1}, \gamma_{2}$ on $T_{0}$ such that both $M\left(\gamma_{1}\right)$ and $M\left(\gamma_{2}\right)$ are annular. Gordon [2] showed that $\Delta\left(\gamma_{1}, \gamma_{2}\right) \leq$ 5 , and furthermore, together with $\mathrm{Wu}[5$, showed that there are only three specific manifolds $M$ realizing $\Delta\left(\gamma_{1}, \gamma_{2}\right)=4$ and 5 . These manifolds are the exteriors of the Whitehead link, the Whitehead sister link and the 2-bridge link corresponding to $3 / 10$ in the 3 -sphere $S^{3}$. Also, he [3, Theorem 5.3] constructed a hyperbolic $k$ component link in $S^{2} \times S^{1}$ for any $k \geq 4$ whose exterior realizes $\Delta\left(\gamma_{1}, \gamma_{2}\right)=2$, and he asked [3, Question 5.3] what is the maximal value for $\Delta\left(\gamma_{1}, \gamma_{2}\right)$ if $M$ is bounded by at least four tori. In this paper, we give an answer to this question.

Theorem 1. Let $M$ be a hyperbolic 3-manifold with $\partial M$ a union of tori. Suppose that there are two slopes $\gamma_{1}$ and $\gamma_{2}$ on a specified boundary torus of $M$ such that

Received by the editors January 27, 2005 and, in revised form, March 21, 2005.

2000 Mathematics Subject Classification. Primary 57M25.

Key words and phrases. Dehn filling, annular manifold.

(C)2006 American Mathematical Society Reverts to public domain 28 years from publication 

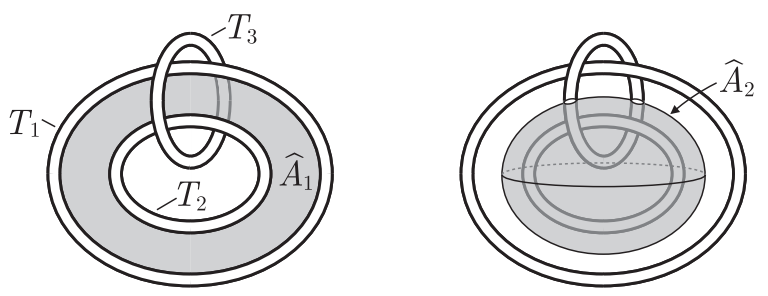

FiguRE 1.

both $M\left(\gamma_{1}\right)$ and $M\left(\gamma_{2}\right)$ are annular. If $\Delta\left(\gamma_{1}, \gamma_{2}\right) \geq 3$, then $\partial M$ is a union of at most three tori.

We remark that there is a hyperbolic 3-manifold with three boundary tori, called the magic manifold, which has two annular Dehn fillings at distance 3. See 4 , Lemma 7.6].

We now go on to the proof of Theorem 1. As mentioned before, if $\Delta\left(\gamma_{1}, \gamma_{2}\right) \geq$ 4 , then $M$ is bounded by only two tori. So, we assume $\Delta=3$ and assume for contradiction that $\partial M$ is a union of at least four tori.

Lemma 2. $M\left(\gamma_{i}\right)$ is homeomorphic to $P \times S^{1}$, where $P$ is a pair of pants.

Proof. Put $X=M\left(\gamma_{i}\right)$. Then $X$ is irreducible by [8, 10], and atoroidal by [7. Since $\partial M$ is a union of at least four tori, $X$ has at least three torus boundary components $T_{1}, T_{2}, T_{3}$.

By assumption $X$ is annular. If $A$ is an essential annulus with boundary components on $T_{1}$ and $T_{2}$, then the frontier of $N\left(A \cup T_{1} \cup T_{2}\right)$ is a torus $T$. Since $X$ is connected, irreducible and atoroidal, $T$ must be parallel to $T_{3}$, hence $X=N\left(A \cup T_{1} \cup T_{2}\right)$ and the result follows. The case that both boundary components of $A$ are on the same torus is similar. We omit the details.

Let $T_{1}, T_{2}, T_{3}$ be the three torus boundary components of $M\left(\gamma_{i}\right)$. Note that $P \times S^{1}$ contains an essential annulus connecting two distinct boundary tori and an essential annulus meeting only one boundary torus.

Let $\widehat{A}_{1}$ be an essential annulus in $M\left(\gamma_{1}\right)$ which has one boundary circle in each of $T_{1}$ and $T_{2}$. Let $\widehat{A}_{2}$ be an essential annulus in $M\left(\gamma_{2}\right)$ whose boundary is contained in $T_{3}$. Note that $\partial \widehat{A}_{1} \cap \partial \widehat{A}_{2}=\emptyset$. Also, notice that $\widehat{A}_{1}$ is non-separating, while $\widehat{A}_{2}$ is separating. See Figure 1 .

We may assume that $\widehat{A}_{1}$ meets the core of $V_{\gamma_{1}}$ transversely. Then $\widehat{A}_{1} \cap V_{\gamma_{1}}$ is a disjoint union of meridian disks $u_{1}, u_{2}, \ldots, u_{n_{1}}$ of $V_{\gamma_{1}}$, numbered successively along $V_{\gamma_{1}}$, and $n_{1}$ is chosen to be minimal among all essential annuli. Similarly, $\widehat{A}_{2}$ intersects $V_{\gamma_{2}}$ in meridian disks $v_{1}, v_{2}, \ldots, v_{n_{2}}$ of $V_{\gamma_{2}}$ ( $n_{2}$ is even). Let $A_{i}=$ $\widehat{A}_{i} \cap M(i=1,2)$ and assume that $A_{1}$ and $A_{2}$ intersect transversely and minimally.

In the usual way [1, 2], the arc components of $A_{1} \cap A_{2}$ define labelled graphs $G_{1}$ on $\widehat{A}_{1}$ and $G_{2}$ on $\widehat{A}_{2}$. The vertices of $G_{i}$ are $u_{1}, u_{2}, \ldots, u_{n_{1}}$ (or $v_{1}, v_{2}, \ldots, v_{n_{2}}$ ), each having a sign according to whether the core of the attached solid torus passes $\widehat{A}_{i}$ from the positive side or the negative side at the vertex. An edge of $G_{i}$ is an arc component of $A_{1} \cap A_{2}$. Since $\partial \widehat{A}_{1} \cap \partial \widehat{A}_{2}=\emptyset$, each edge of $G_{i}$ connects two (possibly the same) vertices of $G_{i}$. If an edge endpoint lies in $\partial u_{x} \cap \partial v_{y}$, then the 
point is labelled $y$ at $u_{x}$ and labelled $x$ at $v_{y}$. An edge is called an $x$-edge if one endpoint is labelled $x$.

An edge in $G_{i}$ is positive if it connects the vertices of the same sign. Otherwise, it is negative. The parity rule [1] says that an edge is positive in one graph if and only if it is negative in the other graph.

Assume that $G_{i}$ has at least two labels. For a label $x, G_{i}^{+}(x)$ denotes a subgraph of $G_{i}$ consisting of all vertices and all positive $x$-edges of $G_{i}$. A disk face in $G_{i}^{+}(x)$ is called an $x$-face. A cycle $\sigma$ in $G_{i}$ consisting of positive edges is a Scharlemann cycle if it bounds a disk face of $G_{i}$, and all the edges in $\sigma$ have the same pair of labels $\{x, x+1\}$ at their endpoints. It is a well-known fact that any $x$-face contains a Scharlemann cycle [6, Proposition 5.1].

Lemma 3. Neither $G_{1}$ nor $G_{2}$ contains a Scharlemann cycle.

Proof. By [10, Lemma 5.4(1)], $G_{2}$ cannot contain a Scharlemann cycle.

Assume that $G_{1}$ contains a Scharlemann cycle with label pair $\{x, x+1\}$ and let $f$ be the disk face bounded by the Scharlemann cycle. Let $H$ be the annulus in $\partial V_{\gamma_{2}}$ cobounded by $\partial v_{x}$ and $\partial v_{x+1}$ such that $H \cap \partial f \neq \emptyset$. Then surgering $\left(\widehat{A}_{2}-\operatorname{Int}\left(v_{x} \cup v_{x+1}\right)\right) \cup H$ along $f$ gives an annulus $A$ in $M\left(\gamma_{2}\right)$ which intersects the core of $V_{\gamma_{2}}$ less than $n_{2}$ times. Since $\partial A=\partial \widehat{A}_{2}, A$ is incompressible. Thus $A$ is boundary-parallel in $M\left(\gamma_{2}\right)$. Then one side of $\widehat{A}_{2}$ cannot contain a component of $\partial M$. This is a contradiction. See Figure 1 .

If $n_{1}=1$, then the edges of $G_{1}$ are mutually parallel. Then $G_{1}$ would contain Scharlemann cycles. Thus $n_{1} \geq 2$. Since $\widehat{A}_{2}$ is separating, $n_{2} \geq 2$.

Lemma 4. Let $\{i, j\}=\{1,2\}$. Then any vertex of $G_{i}$ has at most $n_{j}$ negative edge endpoints.

Proof. Assume, for example, that a vertex $u_{x}$ has more than $n_{2}$ negative edge endpoints. Suppose that $G_{2}^{+}(x)$ has $V$ vertices, $E$ edges, and $F$ disk faces. Then $V=n_{2}, E>n_{2}$ and $F \geq E-V+\chi\left(\widehat{A}_{2}\right)=E-V>0$. Thus $G_{2}$ contains an $x$-face and hence it contains a Scharlemann cycle, contradicting Lemma 3 ,

Let $K_{i}$ be the number of negative edge endpoints in $G_{i}$. Then by Lemma 4 we have $K_{i} \leq n_{1} n_{2}$. Since there is a total of $3 n_{1} n_{2}$ edge endpoints on $G_{1}$, by the parity rule we have $K_{1}=3 n_{1} n_{2}-K_{2}$. Therefore

$$
K_{1}=3 n_{1} n_{2}-K_{2} \geq 3 n_{1} n_{2}-n_{1} n_{2}=2 n_{1} n_{2} .
$$

This gives a contradiction and completes the proof of Theorem 1 .

I thank the referee for helpful comments.

\section{REFERENCES}

1. M. Culler, C. McA. Gordon, J. Luecke, and P.B. Shalen, Dehn surgery on knots, Ann. of Math. 125 (1987), 237-300. MR0881270 (88a:57026)

2. C. McA. Gordon, Boundary slopes on punctured tori in 3-manifolds, Trans. Amer. Math. Soc. 350 (1998), 1713-1790. MR1390037 (98h:57032)

3. C. McA. Gordon, Small surfaces and Dehn filling, Proceedings of the Kirbyfest (Berkeley, CA, 1999), Geom. Topol. Monogr. 2, 177-199. MR1734408 (2000j:57036)

4. C. McA. Gordon and Y.-Q. Wu, Toroidal and annular Dehn fillings, Proc. London Math. Soc. 78 (1999), 662-700. MR1674841 (2000b:57029)

5. C. McA. Gordon and Y.-Q. Wu, Annular Dehn fillings, Comment. Math. Helv. 75 (2000), 430-456. MR1793797 (2001j:57024) 
6. C. Hayashi and K. Motegi, Only single twists on unknots can produce composite knots, Trans. Amer. Math. Soc. 349 (1997), 4465-4479. MR1355073 (98b:57010b)

7. S. Lee, and M. Teragaito, Boundary structure of hyperbolic 3-manifolds admitting annular and toroidal fillings at large distance, to appear in Canad. J. Math.

8. R. Qiu, Reducible Dehn surgery and annular Dehn surgery, Pacific J. Math. 192 (2000), 357-368. MR1744575 (2001b:57036)

9. W. Thurston, Three dimensional manifolds, Kleinian groups and hyperbolic geometry, Bull. Amer. Math. Soc. 6 (1982) 357-381. MR0648524 (83h:57019)

10. Y.-Q. Wu, Sutured manifold hierarchies, essential laminations, and Dehn surgery, J. Diff. Geom. 48 (1998), 407-437. MR.1638025(99h:57043)

School of Mathematics, Korea Institute for Advanced Study, 207-43 CheongryangriDong, Dongdaemun-gu, Seoul 130-722, Korea

E-mail address: slee@kias.re.kr 